\title{
A Customized Reminder and Auto Sending Mobile Application (CRAS)
}

\author{
Rehab Abdullah Alawadh ${ }^{1}$, Arwa Mohammed Almohaimeed ${ }^{2}$, Norah Daifallah Alfuraydi ${ }^{3}$, Ghada Hamad Alkabeer ${ }^{4}$, \\ Ruba Saad Alanber ${ }^{4}$ \\ Department of Information Technology, College of Computer, Qassim University, Buraydah, Saudi Arabia.
}

ORCIDs: 0000-0001-8305-6357 (Rehab), 0000-0002-8550-9011 (Arwa), 0000-0003-2894-0080 (Norah), 0000-0003-4225-7042 (Ghada), 0000-0002-3086-2723 (Ruba)

\begin{abstract}
Nowadays, given the importance of text messaging in everyday and practical life. You may need to send an important message later and forget to send it at the right time. Therefore, a customized reminder and auto sending mobile application which we called (CRAS) system is considered as one of the most important systems at present to facilitate our daily life. This application is quite different from regular messaging or deployed reminder applications. It comes with a simple and user-friendly interface, which allows scheduling messages to be sent at a specific date and time. No more reminders, never miss a message, anniversary celebrations, or an important meeting. Just set the date and time the message was delivered and leave the rest to the application that will send the message on time.
\end{abstract}

Keywords: Scheduled message, Text message, Scheduling system.

\section{INTRODUCTION}

Based on our research, scheduling applications has been available since 2015 until this day. Most of these apps schedule the message based on SMS only, where the rest of these apps based on multiple cross-platforms, like (WhatsApp, Emails, Facebook, GroupMe...and more.) The history behind the creation of scheduled messages apps was initially a reminder system for appointments rather than making phone calls, which takes time. The concept of messages scheduling was then touched upon, and these applications branched out in scheduling messages through social media. Nowadays, as we are busy with our businesses, and there are no applications doing the whole sending process automatically, so in this paper we proposed our system a customized reminder and auto sending mobile application which we called (CRAS) that will facilitate many operations of daily life and business processes. The customized reminder and auto sending mobile application is quite different from regular messaging or deployed reminder applications. It comes with a simple and user-friendly interface, which allows scheduled messages and events to be sent at a specific date and time. No need for more reminders and never misses a message, anniversary celebrations, or important meetings. Just set the date and time the message was delivered and leave the rest to the application that will send the message on time.

\section{LITERATURE REVIEW}

This paper aims to develop a customized reminder and auto sending mobile application where messages service considered as one of the essential services that have been used since 1992 until now [1].

Text messages or Texting is the act of sending short electronic messages using cell phones, usually using SMS. Texting or SMS is a way of communication. It may be sent among cell phones - or from a PC or handheld to a cell phone. The" short" part comes from the maximum size of the text messages, which is 160 characters (alphabetic and numeric) Moreover, it has grown to include multimedia messages known as MMS, that contain digital videos, images, sound, and emojis [2].

Being able to send short, written messages via mobile phone has developed in several methods. The most significant benefit of texting is that it allows the users to communicate instantly with other people anywhere, but without interrupting them in the way that a phone call would. Text messaging became a universal feature for phones, making it more affordable in an unlimited package. Today, SMS is the most widely-used data application in the world, with $81 \%$ of mobile phone subscribers using it. SMS importance become more than chatting with friends. It also lets users receive updates and alerts. Keep track of the finances, send an email, and much more [3]. The birth of texting was a savior for everybody. It is not just made life easier in terms of communication or entertainment but has done so in various fields of life!

There are some issues in the currently related available applications, which summarized as below:

- The Sending Process: Some applications working way are rather annoying and scary; The sending process does not run in the background; it disables the user processes and starts operates in the foreground.

- Device Password Issues: Requiring to remove the device password to use the application services is one of the most important problems, that may not get the acceptance of users.

- Registration: Restrict users to register to be able to explore the application, and be able to use its services, may not meet the acceptance of the users! Where most users need to explore the application services before registration. Avoiding this kind of problem can be done with the "Skip Registration" option. 


\section{CRAS PROPOSED SYSTEM}

The proposed application has been developed in Java programming language using the Android Studio program to solve related previous problems in term of "Sending automatically" and provide missing features and services. The primary function of the app is to be able to schedule SMS messages and send them automatically without user intervention, also to be able to schedule email messages with alerts feature. Plus, the ability to create custom events for the user and schedule them to send as SMS messages. It has also developed to be easy-to-use for all categories, as well as the ease-of-access.

\section{III.I PROPOSED MODEL}

The proposed model of this system summarized as below:

- Registration: The user enters their username, number, email and password to be saved in the system.

- New scheduled message: The creation of a new scheduled message to be sent as an SMS message, or an email message. The message will contain receiver information, content and the specific date and time.

- Scheduled Messages: This page contains the scheduled messages to be sent automatically.

- Calendar: This page contains a calendar in addition to specific events, which are [Saudi Events, Qassim University Events, and User Customized Events].

- Settings: This page contains the user information.

\section{METHODOLOGY APPROACH}

Software Development Life Cycle SDLC is an approach applied in software engineering to explain procedures for planning, building, coding, testing, and implementation of a user requirement specification. The systems development lifecycle applies to a range of hardware and software configurations. SDLC is a step-by-step operation for building quality software for users. It includes different phases that are done one by one, which is necessary for software developers, like planning, analyzing, designing, coding, and implementation. It includes an evaluation of current software, data collection, and feasibility study and request acceptance [4].

SDLC has different types of SDLC methodologies, including the agile SDLC mode. The agile model is a mixture of the iterative and incremental processes. It focuses on process adaptability and user gratification by the fast delivery of working software products. The agile approach breaks the software product into a small incremental build. These incremental builds are finished in iterations. Where in each iteration, the development team works on the planning, requirements gathering and analyzing, design, implementation, testing, and acceptance testing. At the ends of iteration, a working product is delivered to the users and stakeholders [5]. Fig 1 shows the SDLC of A Customized Reminder and Auto Sending Mobile Application (CRAS).

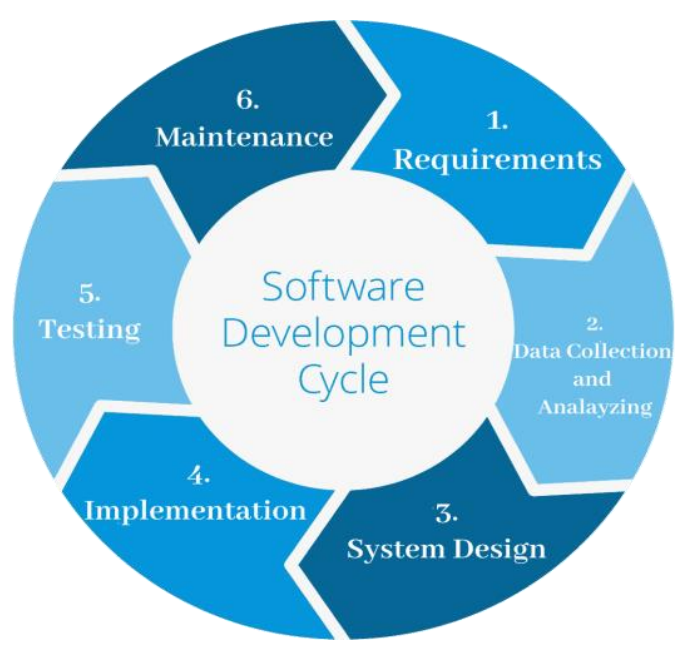

Fig. 1 System Development Life Cycle.

\section{IV.I DATA COLLECTION AND ANALYSIS}

\section{IV.I.I DATA COLLECTION}

Data collection is a process of gathering information from all the relevant sources to gain answers to the study problem and evaluate the results [6]. In order to collect the data that helps this study, a questionnaire was used to find out how much users need this app and how satisfied they are with similar apps currently available.

The questionnaire included five questions of multiple-choice type with two labels (Yes, No) as follows:

- Question 1: Are you an academic employee.

- Question 2: Have you ever forgotten to send an important message.

- Question 3: Do you feel the importance of having a program schedule your messages and sent them automatically.

- Question 4: Have you ever used an application to schedule messages.

- Question 5: If yes, how satisfied are you with this app.

\section{IV.I.II DATA ANALYSIS}

Data Analysis is the process of systematically utilizing statistical and logical techniques to represent and explain, condense and recap, and evaluate data [7]. The data analysis uses to sort, organize, store, process, analyze, and study the collected data to discover new knowledge [8].

During this phase, 232 responses from the questionnaire were collected, checked, and cleaned by google sheets software. 


\section{The needs of the app based on people who forget their messages}

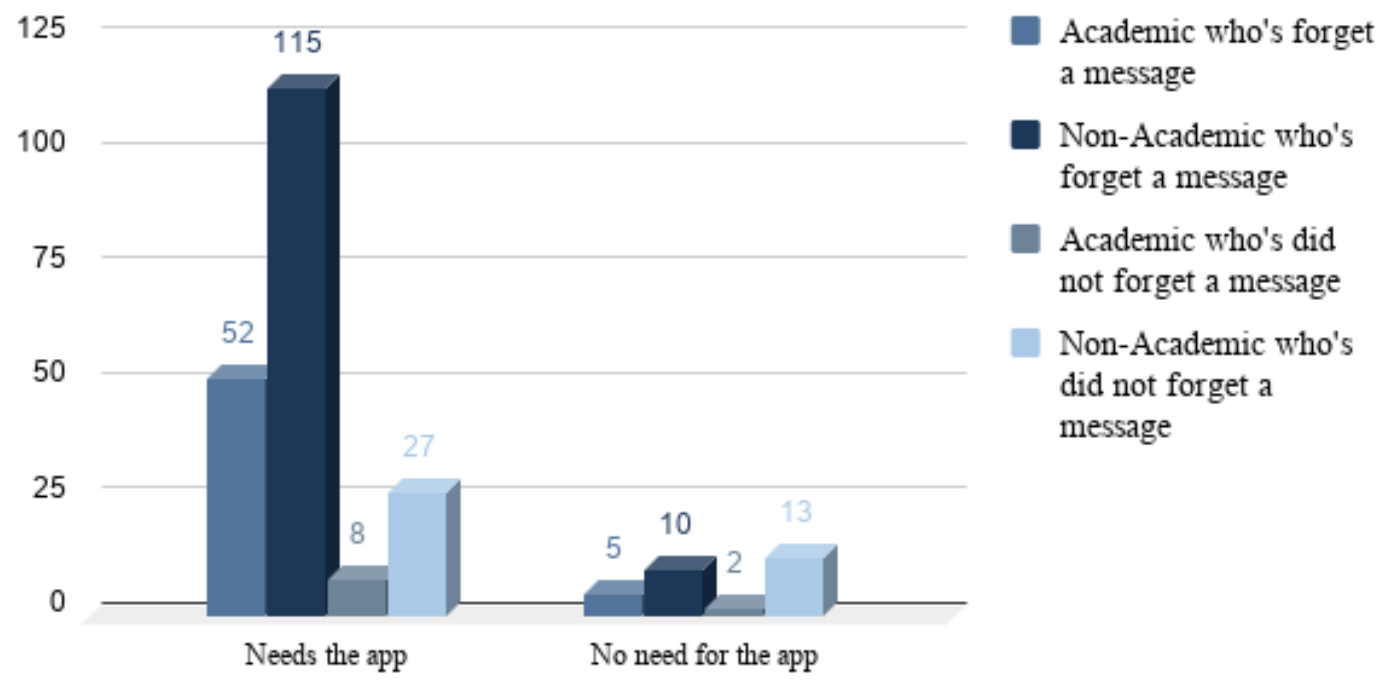

Fig. 2 The needs of the app based on people who forget their messages.

As shown in Fig 2, based on the responses in the previous questionnaire, and the analysis of the data obtained, the audience's need for this application was discovered as follows:

Responsive academic employees represent 67 persons, which is $28.9 \%$ of the total respondents for the questionnaire. 60 academic employee needs the proposed application, which is $89.6 \%$ of the total academic employees, while 7 of the responsive academic employees do not needs the proposed application, which is $10.4 \%$ of the total academic employees. On the other hand, the responsive of non-academic were 165 persons, which is $71.1 \%$ of the total respondents for the questionnaire. A 142 non-academics need the proposed application, which is $86.1 \%$ of the total non-academic persons, while 23 persons of the responsive non-academic did not need the proposed application, which is $13.9 \%$ of the total nonacademic persons.

Data Analysis of this study can be summarized as:

The total responses for the questionnaire were 232 response, $28.9 \%$ of them represent Academic employees, while $71.1 \%$ of them represents Non-academic people (ordinary people) $89.6 \%$ of the academic employees need the proposed application, which represents $25.8 \%$ of the total respondents for the questionnaire. While $86.1 \%$ of the non-academic persons (ordinary people) need the proposed application, which represents $61.2 \%$ of the total respondents for the questionnaire. The total responsive who needs the proposed application represents $87 \%$ of the total responses, which represents 202 respondents of 232 .

\section{CRAS SYSTEM DESIGN PROCEDURE}

The software paths and functionality are designed with tools and diagrams according to the requirements studied in the first stage. To help determine the structure of the system as whole. The input determines the points that work to improve the detected negatives. The output will be to reduce the forgotten messages in personal and social communities and facilitating daily operations. The Data Flow Diagram DFD is an analysis and design method used in software engineering [9]. It is a visible tool that shows the transformation of the data in a system. DFD has techniques to model the data flow, which aids explain details of the data flows and processes. It offers a symbol system to represent data flow to describe a system at various levels. Fig. 3 shows the DFD. 


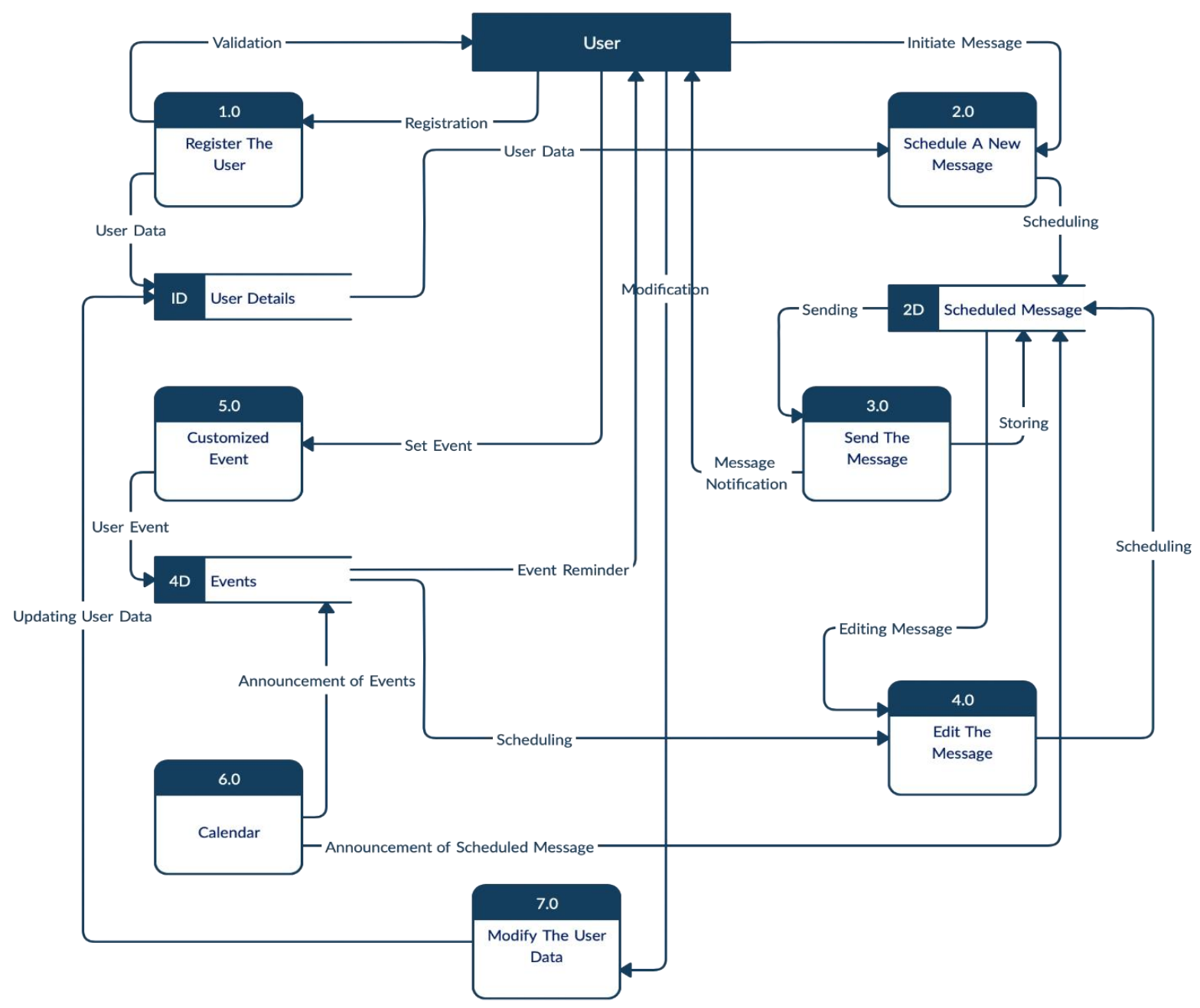

Fig. 3 The DFD of The Proposed System.

\section{SYSTEM IMPLEMENTATION}

The first interface is the Welcome Interface, which is shown when the user enters the application. This interface provides three options as buttons, which are a sign-in button that directs the user to the sign-in interface, a sign-up button that directs the user to the sign-up interface, and a skip button that directs the user to the Homepage interface. Fig. 4 shows the welcome interface.

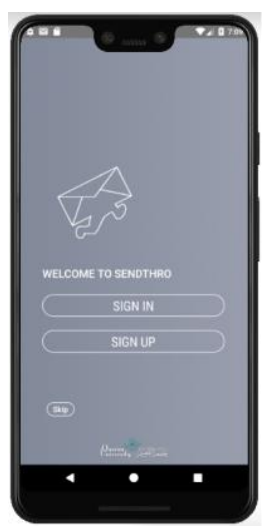

Fig. 4 The Welcome Interface.
The scheduled message interface provides two buttons, which are an SMS scheduler button that directs the user to the SMS scheduler interface, and an email scheduler button that directs the user to the email scheduler interface. Also, it provides an animated button with two sub-buttons, which are creating an SMS scheduled message, and create an email scheduled message to provide the ease-of-access to the users. Fig. 5 shows the scheduled message interface.

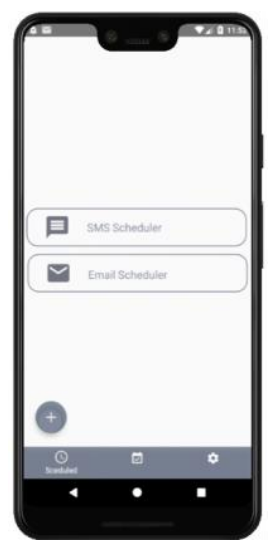

Fig. 5 The Scheduled Message Interface. 
The calendar and events interface comes with a top navigation bar consist of a calendar button and events button to facilitate the navigating between calendar interface and events interface. The calendar interface provides a calendar view that is clickable to provide the ability to add new events on a specific day, as well as a text view that is clickable to direct the user to the create a new event interface. Fig 6 shows the calendar and events interface.

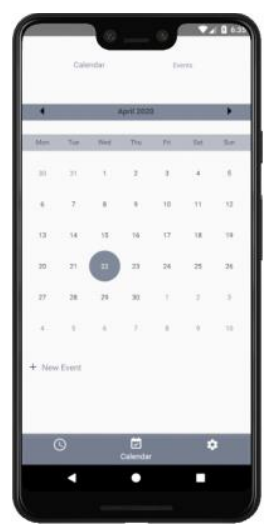

Fig. 6 The Calendar and Events Interface.

The events interface comes with a list view that is clickable, which consists of user events that direct the user to the user events interface, Saudi events that direct the user to the Saudi events interface, and Qassim University events that direct the user to the Qassim University events interface. Fig 7 shows the events interface.

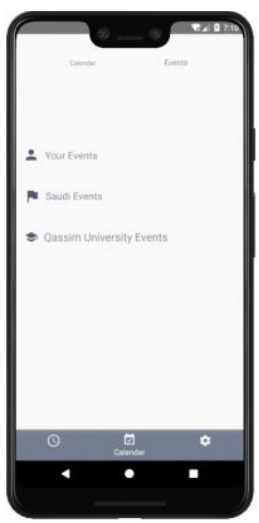

Fig. 7 The Events Interface.

On the calendar interface, by clicking either the text view that appears down the calendar or on a specific day, a create a new event interface appears. On the create a new event interface, the event title, date, time, repeat option, repeat interval, and notification button is required to schedule the event. By clicking on the check button, the event is saved as a list in the user events interface. Fig 8 shows the create a new event interface.

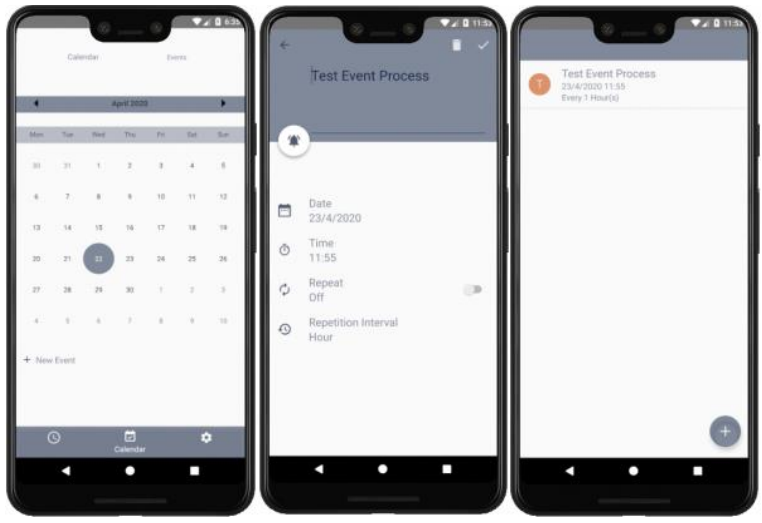

Fig. 8 Create a New Event Interface.

The user is alerted when it is the event time with a notification, which contains a button to schedule the event as an SMS message. By clicking on the Schedule This Event button, the create a new SMS scheduled message interface appears. Fig 9 shows the user event notification.
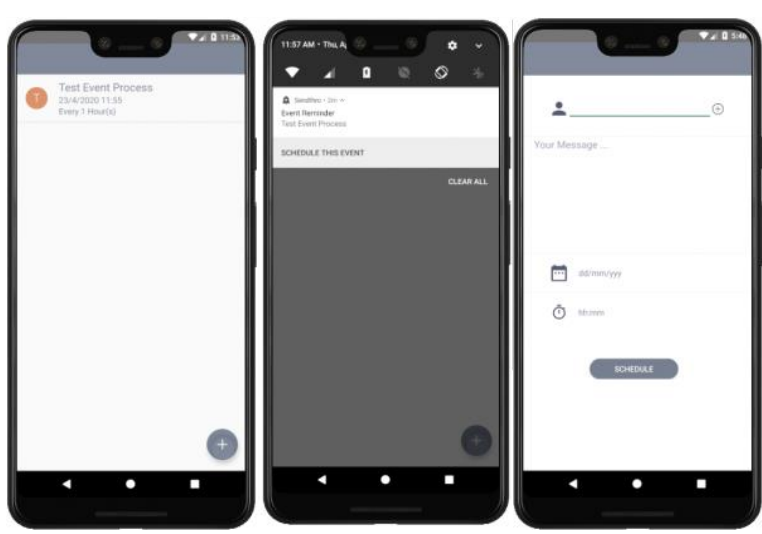

Fig. 9 User Event Notification.

On the create a new SMS scheduled message interface, the receiver number, message content, date, and time are required to schedule the SMS message. The system uses the SIM-card mobile number as sender number. By clicking on the schedule button, the message is saved as a list in the SMS scheduler interface. The user is alerted if the scheduled message is sent with notification, which contains the receiver number. The SMS scheduled message has been sent successfully. Fig 10 shows the Create a New SMS Scheduled Message Interface. 

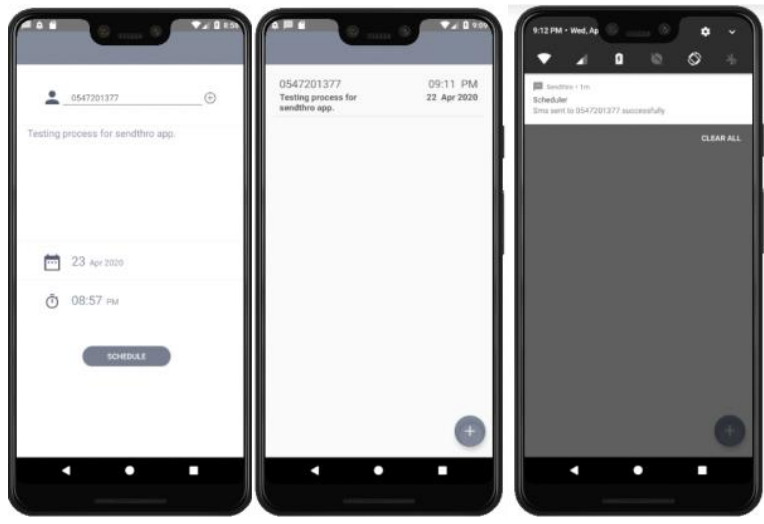

Fig. 10 Create a New SMS Scheduled Message Interface.

\section{DISCUSSION RELATED TO PROPOSED WORK}

For such applications, database usage is a significant part of data storage. Therefore, the development process relied on two methods of storing data in the database, the user's login data stored in the Firebase Cloud Fire-store database. In contrast, the data related to messages and special events are stored in a local database using the SQLite database. The application was provided with some specifications to raise the level of security, where the messages created by the application and events stored in the local database, which cannot be accessed using another device. Also, the password change process cannot be performed Unless there is a new login status for the user. Moreover, increasing the level of usability lies with the navigation bar, in addition to data integrity, which can be ensured by using Firebase and SQLite.

\section{CONCLUSION AND FUTURE WORK}

In this study, an application developed on the Android platform, which relies on the auto sending of scheduled messages at a specific date and time as SMS messages. The application offers annual events for both Kingdom of Saudi Arabia events and Qassim University events. In addition to user-customized events and the ability to schedule them to send as SMS messages.

In future updates to this application, the scheduling process will be developed to support automatically sent to both email and WhatsApp. This application will also be upgraded to be provided on both Android and iOS platforms. The application upgrade has been started to run on a cross-platform using the React-Native framework to be the next version of the app.

\section{REFERENCES}

1. F. Trosby, K. Holley, and I. Harris, Short message service (SMS): the creation of personal global text messaging. John Wiley \& Sons, 2010.

2. C. Erickson. (2012, Sep) A brief history of text messaging. Accessed:17-sep-2019. [Online]. Available: https://mashable. com/2012/09/21/text-messaging-history/
3. Sms and mms defined. Accessed:17-sep-2019. [Online]. Available: https://help.textline.com/hc/en-us/ articles/360003942351-SMS-and-MMS-Defined

4. M. K. Sharma, "A study of sdlc to develop well engineered software,"International Journal of Advanced Research in Computer Science, vol. 8, no. 3, 2017.

5. K. Ali, "A study of software development life cycle process models,"International Journal of Advanced Research in Computer Science, vol. 8, no. 1, pp. 15 - 23, 2017.

6. Data collection methods - research-methodology. Accessed: 24-nov-2019. [Online]. Available:https://researchmethodology.net/research-methods/data-collection/

7. Data analysis. Accessed:24-nov-2019. [Online]. Available: https://ori.hhs.gov/education/products/n_illinois_u/ datamanagement/datopic.html

8. J. Chen, Q. Jiang, Y. Wang, and J. Tang, "Study of data analysis model based on big data technology," in 2016 IEEE International Conference on Big Data Analysis (ICBDA), March 2016, pp. 1-6

9.Q. Li and Y.-L. Chen,Data Flow Diagram. Berlin, Heidelberg: Springer Berlin Heidelberg,2009, pp. 85-97. [Online]. Available:https://doi.org/10.1007/978-3-54089556-5 4 